\title{
FOLIAR APPLICATION OF CHITOSAN ON GROWTH AND YIELD ATTRIBUTES OF MUNGBEAN (VIGNA RADIATA (L.) WILCZEK)
}

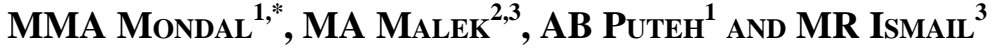 \\ Crop Physiology Division, Bangladesh Institute of Nuclear Agriculture, \\ Mymensingh, Bangladesh
}

Key words: Foliar spray, Chitosan, Mungbean, Seed yield

\begin{abstract}
Plant parameters such as plant height, branch and leaf number/plant, leaf area/plant, total dry mass/plant, photosynthesis, harvest index, chlorophyll, nitrate reductase and number of pods/plant increased significantly with the increasing concentration of chitosan up to $50 \mathrm{ppm}$. It resulted the highest seed yield in mungbean.

Mungbean, Vigna radiata (L.) Wilczek, is one of the most important pulses in south-east Asia. However, its yield is much lower than that of other legume crops such as grasspea, chickpea and lentil (FAO 2007). Flower and pod abortion is a common phenomenon in mungbean and abscission of a large proportion of reproductive structures $(69-90 \%)$ before maturity, would be the primary cause of lowering yield in mungbean (Fakir et al. 2011). There are reports that application of growth regulators reduce abscission and increase yield components in soybean and tomato (Nahar and Ikeda 2002, Imam et al. 2010).

A few researches were carried on the effects of chitosan on plant growth and development and its productivity (Patkowska et al. 2006 , Sereih et al. 2007, Gornik et al. 2008). Recently, chitosan enhanced plant growth and development have been reported Chibu et al. (2002), Mondal et al. (2012). So far our knowledge to chitosan induced growth, yield attributes and fruit yield of mungbean is scanty. So, the present research was undertaken to study the effects of chitosan on morphological features, yield attributes and yield in mungbean under sub-tropical climate.

Two varieties of mungbean, namely BINAmung-7 and BINAmung-8 obtained from BINA were used for both pot and field experiments during Kharif-I season (March-May) of 2010 and 2011. Five concentrations of chitosan viz., 0, 25, 50, 75 and $100 \mathrm{ppm}$ were applied twice at 25 (4 - 5 leaves stage) and 35 days after sowing (DAS), just before flowering, by a hand sprayer in the afternoon. For pot culture, the soil was thoroughly mixed with urea, triple superphosphate, muriate of potash and gypsum at the rate of 2.0, 4.0, 3.0 and $2.0 \mathrm{~g} / \mathrm{pot}$ corresponding to $40,80,60$ and $40 \mathrm{~kg} / \mathrm{ha}$, respectively. Total amount of urea, TSP, MP and gypsum were applied as basal dose during soil preparation. The pots $(30 \times 25 \mathrm{~cm})$ were filled with $10 \mathrm{~kg}$ of soil. The pot experiment was laid out at completely randomized design with four replications. Five seeds were sown in each pot and 15 DAS, they were thinned to two seedlings. The field experiment was also laid out at randomized complete block design with three replications. The unit plot size was $3 \times 2 \mathrm{~m}$. The row to row and plant to plant distances were 30 and $10 \mathrm{~cm}$, respectively. Intercultural operations such as irrigation, weeding, mulching and pest control were followed as and when necessary.
\end{abstract}

\footnotetext{
*Author for correspondence: <ma_mondol@yahoo.com>. ${ }^{1}$ Department of Crop Science, Faculty of Agricuture, Universiti Putra Malaysia, 43400 UPM Serdang, Selangor Darul Ehsan, Malaysia. ${ }^{2}$ Plant Breeding Division, Bangladesh Institute of Nuclear Agriculture, Mymensingh, Bangladesh. ${ }^{3}$ Institute of Tropical Agriculture, Universiti Putra Malaysia, 43400 UPM Serdang, Selangor Darul Ehsan, Malaysia
} 
Flower counting (five plants for each replication) was made each day starting from the date of opening of first flower until flowering ceased. Chlorophyll content in leaves, nitrate reductase (NR) activity in leaves and photosynthesis in leaf were determined at 45 - 50 DAS (flowering and fruiting stage) of upper $2-3$ nodal leaves in the canopy. Chlorophyll was determined following Yoshida et al. (1976). NRase was estimated following Stewart and Orebamjo (1979). Photosynthesis was measured by automatic photosynthesis meter (LICOR 6400XT, USA). Leaf area, yield and yield components, dry matter production and it's partitioning into plant parts were determined at harvest. Harvest index was calculated from the data using formula: (Economic yield/biological yield) $\times 100$. Plant material was oven dried at $80 \pm 2^{\circ} \mathrm{C}$ for $72 \mathrm{hrs}$. The total dry matter/plant was estimated by summing dry matter of leaves, stem and roots per plant. The data were analyzed using Microsoft Statistical Data Management Package (MSTAT) program.

Results show that plant height, branch and leaf number and LA/plant both at pot and field conditions increased with the increasing chitosan concentration till $50 \mathrm{ppm}$ (Table 1). The higher plant height, branch and leaf number and LA/plant were recorded in between 50 and $100 \mathrm{ppm}$ of chitosan. Leaf number and LA were greater in chitosan treated plants than control might be due to increased number of branches/plant (Table 1). These results are in conformity in tomato and okra (El-Tantawy 2009 and Mondal et al. (2012).

Table 1. Effect of chitosan on growth attributes of mungbean.

\begin{tabular}{|c|c|c|c|c|c|c|c|c|}
\hline \multirow[t]{2}{*}{$\begin{array}{l}\text { Treatments } \\
\text { conc. (ppm) }\end{array}$} & \multicolumn{2}{|c|}{$\begin{array}{l}\text { Plant height } \\
\text { (cm) }\end{array}$} & \multicolumn{2}{|c|}{$\begin{array}{l}\text { Branches/ } \\
\text { plant (no.) }\end{array}$} & \multicolumn{2}{|c|}{$\begin{array}{c}\text { Leaves/ } \\
\text { plant (no.) }\end{array}$} & \multicolumn{2}{|c|}{$\begin{array}{c}\text { Leaf area/ } \\
\text { plant }\left(\mathrm{cm}^{2}\right)\end{array}$} \\
\hline & Pot & Field & Pot & Field & Pot & Field & Pot & Field \\
\hline 0 & $31.1 \mathrm{c}$ & $37.3 \mathrm{~b}$ & $1.85 \mathrm{c}$ & $2.00 \mathrm{~b}$ & $12.6 \mathrm{~d}$ & $15.2 \mathrm{c}$ & $1056 \mathrm{c}$ & $1265 \mathrm{~b}$ \\
\hline 25 & $34.3 \mathrm{~b}$ & $38.1 \mathrm{ab}$ & $2.25 \mathrm{~b}$ & $2.15 \mathrm{~b}$ & $14.0 \mathrm{c}$ & $16.5 \mathrm{bc}$ & $1165 \mathrm{~b}$ & $1305 \mathrm{ab}$ \\
\hline 50 & $35.4 \mathrm{ab}$ & $38.7 \mathrm{ab}$ & $2.65 \mathrm{a}$ & $2.25 \mathrm{ab}$ & $17.1 \mathrm{a}$ & $17.6 \mathrm{ab}$ & $1255 \mathrm{a}$ & $1295 \mathrm{ab}$ \\
\hline 75 & $36.8 \mathrm{a}$ & $39.0 \mathrm{a}$ & $2.57 \mathrm{a}$ & $2.50 \mathrm{a}$ & $15.5 \mathrm{~b}$ & $18.2 \mathrm{a}$ & $1220 \mathrm{ab}$ & $1390 \mathrm{a}$ \\
\hline 100 & $35.8 \mathrm{ab}$ & $39.4 \mathrm{a}$ & $2.65 \mathrm{a}$ & $2.50 \mathrm{a}$ & $16.6 \mathrm{a}$ & $18.7 \mathrm{a}$ & $1241 \mathrm{a}$ & $1340 \mathrm{a}$ \\
\hline F test & $* *$ & $*$ & $* *$ & $*$ & $* *$ & $* *$ & $* *$ & $*$ \\
\hline \multicolumn{9}{|l|}{ Variety } \\
\hline BINA mung-7 & $39.8 \mathrm{a}$ & $44.5 \mathrm{a}$ & $3.65 \mathrm{a}$ & $3.32 \mathrm{a}$ & $21.1 \mathrm{a}$ & $22.4 \mathrm{a}$ & $1505 \mathrm{a}$ & $1606 \mathrm{a}$ \\
\hline BINA mung-8 & $29.6 \mathrm{~b}$ & $32.5 \mathrm{~b}$ & $1.14 \mathrm{~b}$ & $1.24 \mathrm{~b}$ & $9.23 \mathrm{~b}$ & $12.0 \mathrm{~b}$ & $872 \mathrm{~b}$ & $1019 \mathrm{~b}$ \\
\hline F Test & $* *$ & $* *$ & $* *$ & $* *$ & $* *$ & $* *$ & $* *$ & $* *$ \\
\hline CV (\%) & 4.08 & 4.64 & 7.87 & 11.56 & 5.96 & 7.92 & 5.36 & 7.22 \\
\hline
\end{tabular}

In a column, within treatment, figures having same letter(s) do not differ significantly at $\mathrm{p} \leq 0.05$.

$*, * *$ indicate significant at 5 and $1 \%$ level of probability, respectively.

Total dry mass (TDM)/plant, photosynthesis, chlorophyll and NR increased significantly with the increasing chitosan concentration till $50 \mathrm{ppm}$ (Table 2). The harvest index (HI) increased up to $50 \mathrm{ppm}$ chitosan indicated that dry matter partitioning was in favor of economic yield in mungbean. These results are in consistent with Khan et al. (2002) and El-Tantawy (2009) who reported that application of chitosan increased photosynthesis in leaves of maize and soybean as well as tomato, respectively.

In the present investigation, the number of flowers/plant increased with the increasing chitosan concentration till $75 \mathrm{ppm}$ followed by slightly declining whereas the number of pods/plant increased significantly till $50 \mathrm{ppm}$ (Table 3). Pod length, number of seeds/pod and 1000-seed weight was apparently increased in chitosan treated plants over control plants (Table 3 ). Compared to 50 and $75 \mathrm{ppm}$ chitosan, all yield attributes lowered down at $100 \mathrm{ppm}$ chitosan indicating its toxic effect at higher concentration. 
The highest seed yield/plant both at pot and field condition was at $50 \mathrm{ppm}(8.16$ and 9.31 $\mathrm{g} /$ plant for pot and field, respectively) followed by $75 \mathrm{ppm}(7.66$ and $8.70 \mathrm{~g} / \mathrm{plant}$ for pot and field, respectively), Table 4 . The yield of seeds was higher at $50 \mathrm{ppm}$ chitosan due to increased production of pods/plant (25.4 and 27.1/plant for pot and field, respectively) and superior dry matter partitioning to economic yield (Table 2). Considering yield performance in unit area basis, the higher seed yields were recorded at 50 and $75 \mathrm{ppm}$ chitosan with being the highest at $75 \mathrm{ppm}$ (1913 kg/ha). Chibu et al. (2002) reported that application of chitosan at early stages increased plant growth and development thereby increased the seed yield in rice and soybean.

Table 2. Effect of chitosan on some physiological and biochemical parameters of mungbean.

\begin{tabular}{|c|c|c|c|c|c|c|c|}
\hline \multirow{3}{*}{$\begin{array}{l}\text { Treatments } \\
\text { conc. }(\mathrm{ppm})\end{array}$} & \multicolumn{5}{|c|}{ Physiological parameters } & \multicolumn{2}{|c|}{ Biochemical parameters } \\
\hline & \multicolumn{2}{|c|}{$\begin{array}{l}\text { Total dry mass/ } \\
\text { plant }(\mathrm{g})\end{array}$} & \multicolumn{2}{|c|}{$\begin{array}{c}\text { Harvest } \\
\text { index } \\
(\%)\end{array}$} & \multirow{2}{*}{$\begin{array}{l}\text { Photosynthesis } \\
(\mu \mathrm{mol} \\
\left.\mathrm{CO}_{2} / \mathrm{s} / \mathrm{m}^{2}\right) \\
\text { Field }\end{array}$} & \multirow{2}{*}{$\begin{array}{l}\begin{array}{l}\text { Chlorophyll } \\
(\mathrm{mg} / \mathrm{g} / \mathrm{FW})\end{array} \\
\text { Field }\end{array}$} & \multirow{2}{*}{$\begin{array}{l}\mathrm{NR}(\mu \mathrm{mol} \\
\mathrm{NO}_{2}^{-} \\
/ \mathrm{g} / \mathrm{FW}) \\
\text { Field }\end{array}$} \\
\hline & Pot & Field & Pot & Field & & & \\
\hline 0 & $13.8 \mathrm{c}$ & $15.3 \mathrm{~b}$ & $34.9 \mathrm{~b}$ & $31.5 \mathrm{ab}$ & $15.36 \mathrm{~b}$ & $2.19 \mathrm{~b}$ & $3.84 \mathrm{~b}$ \\
\hline 25 & $14.9 \mathrm{~b}$ & $15.8 \mathrm{~b}$ & $37.2 \mathrm{a}$ & $33.0 \mathrm{ab}$ & $16.71 \mathrm{a}$ & $2.29 \mathrm{ab}$ & $4.21 \mathrm{a}$ \\
\hline 50 & $15.8 \mathrm{ab}$ & $17.4 \mathrm{a}$ & $37.5 \mathrm{a}$ & $33.3 \mathrm{a}$ & $17.29 \mathrm{a}$ & $2.35 \mathrm{a}$ & $4.30 \mathrm{a}$ \\
\hline 75 & $16.6 \mathrm{a}$ & $17.9 \mathrm{a}$ & $34.5 \mathrm{~b}$ & $32.9 \mathrm{ab}$ & $17.25 \mathrm{a}$ & $2.35 \mathrm{a}$ & $4.29 \mathrm{a}$ \\
\hline 100 & $16.0 \mathrm{a}$ & $17.5 \mathrm{a}$ & $34.5 \mathrm{~b}$ & $30.8 \mathrm{~b}$ & $17.41 \mathrm{a}$ & $2.40 \mathrm{a}$ & $4.33 \mathrm{a}$ \\
\hline F test & $* *$ & $* *$ & $*$ & $*$ & $* *$ & $*$ & $* *$ \\
\hline \multicolumn{8}{|l|}{ Variety } \\
\hline BINA mung-7 & $18.2 \mathrm{a}$ & $19.5 \mathrm{a}$ & $32.4 \mathrm{~b}$ & 30.7 & $15.99 \mathrm{~b}$ & $2.21 \mathrm{~b}$ & $4.06 \mathrm{~b}$ \\
\hline BINA mung- 8 & $12.6 \mathrm{~b}$ & $14.1 \mathrm{~b}$ & $39.0 \mathrm{a}$ & 33.9 & $17.61 \mathrm{a}$ & $2.42 \mathrm{a}$ & $4.33 \mathrm{a}$ \\
\hline F Test & $* *$ & $* *$ & $* *$ & $*$ & $* *$ & $* *$ & $* *$ \\
\hline CV (\%) & 6.40 & 6.85 & 5.87 & 5.29 & 5.56 & 5.04 & 4.28 \\
\hline
\end{tabular}

In a column, within treatment, figures having same letter(s) do not differ significantly at $\mathrm{p} \leq 0.05$.

$*, * *$ indicate significant at 5 and $1 \%$ level of probability, respectively.

Table 3. Effect of chitosan on yield attributes of mungbean.

\begin{tabular}{|c|c|c|c|c|c|c|c|c|c|}
\hline \multirow{2}{*}{$\begin{array}{l}\text { Treat- } \\
\text { ments } \\
\text { conc. } \\
\text { (ppm) }\end{array}$} & \multirow{2}{*}{$\begin{array}{c}\begin{array}{c}\text { Flowers/ } \\
\text { plant(no.) }\end{array} \\
\text { Field }\end{array}$} & \multicolumn{2}{|c|}{$\begin{array}{c}\text { Pods/ } \\
\text { plant (no.) }\end{array}$} & \multicolumn{2}{|c|}{$\begin{array}{c}\text { Pod length } \\
(\mathrm{cm})\end{array}$} & \multicolumn{2}{|c|}{$\begin{array}{c}\text { Seeds/pod } \\
\text { (no.) }\end{array}$} & \multicolumn{2}{|c|}{$\begin{array}{l}\text { 1000-seed } \\
\text { weight }(\mathrm{g})\end{array}$} \\
\hline & & Pot & Field & Pot & Field & Pot & Field & Pot & Field \\
\hline 0 & $43.2 \mathrm{~b}$ & $19.9 \mathrm{c}$ & $23.4 \mathrm{~b}$ & 7.54 & 7.62 & 10.26 & 10.80 & $37.26 \mathrm{~b}$ & 35.70 \\
\hline 25 & $43.7 \mathrm{~b}$ & $22.8 \mathrm{~b}$ & $25.1 \mathrm{ab}$ & 7.59 & 7.87 & 10.30 & 11.21 & $37.87 \mathrm{a}$ & 36.10 \\
\hline 50 & $46.9 \mathrm{a}$ & $25.4 \mathrm{a}$ & $27.1 \mathrm{a}$ & 7.59 & 7.90 & 10.40 & 11.30 & $38.00 \mathrm{a}$ & 36.40 \\
\hline 75 & $49.3 \mathrm{a}$ & $23.7 \mathrm{~b}$ & $27.3 \mathrm{a}$ & 7.64 & 7.90 & 10.52 & 11.20 & $37.65 \mathrm{ab}$ & 36.20 \\
\hline 100 & $48.1 \mathrm{a}$ & $22.0 \mathrm{~b}$ & $25.6 \mathrm{ab}$ & 7.59 & 7.82 & 10.22 & 11.27 & $36.80 \mathrm{c}$ & 35.65 \\
\hline$F$ test & $* *$ & $* *$ & $*$ & NS & NS & NS & NS & $* *$ & NS \\
\hline \multicolumn{10}{|l|}{ Variety } \\
\hline $\begin{array}{l}\text { BINA } \\
\text { mung-7 }\end{array}$ & $66.2 \mathrm{a}$ & $29.9 \mathrm{a}$ & $34.7 \mathrm{a}$ & $7.00 \mathrm{~b}$ & $7.21 \mathrm{~b}$ & $10.04 \mathrm{~b}$ & $10.81 b$ & $29.71 b$ & $28.90 \mathrm{a}$ \\
\hline $\begin{array}{l}\text { BINA } \\
\text { mung-8 }\end{array}$ & $26.3 \mathrm{~b}$ & $15.6 \mathrm{~b}$ & $16.8 \mathrm{~b}$ & $8.18 \mathrm{a}$ & $8.44 \mathrm{a}$ & $10.65 \mathrm{a}$ & $11.50 \mathrm{a}$ & $45.32 \mathrm{a}$ & $43.12 b$ \\
\hline F Test & $* *$ & $* *$ & $* *$ & $* *$ & $* *$ & $* *$ & $* *$ & $* *$ & $* *$ \\
\hline $\mathrm{CV}(\%)$ & 5.40 & 7.28 & 8.38 & 1.77 & 2.96 & 3.71 & 4.07 & 1.70 & 3.16 \\
\hline
\end{tabular}

In a column, within treatment, figures having same letter(s) do not differ significantly at $\mathrm{p} \leq 0.05$.

$*, * *$ indicate significant at 5 and $1 \%$ level of probability, respectively; NS = Not significant. 
Among the varieties, BINAmung-7 showed superiority in plant height, branch and leaf production, TDM production, flower and pod production and seed yield over BINAmung-8 whereas it showed superiority in photosynthesis, chlorophyll, NR, pod and seed size and HI than BINAmung-7 (Tables 1- 4).

Table 4. Effect of chitosan on seed yield and interaction of variety and chitosan concentration on seed yield of mungbean.

\begin{tabular}{llllllll}
\hline \multirow{2}{*}{$\begin{array}{l}\text { Treatments } \\
\text { conc. }(\mathrm{ppm})\end{array}$} & \multicolumn{2}{c}{$\begin{array}{c}\text { Seed weight/ } \\
\text { plant }(\mathrm{g})\end{array}$} & $\begin{array}{l}\text { Seed yield } \\
(\mathrm{kg} / \mathrm{ha})\end{array}$ & $\begin{array}{c}\text { Interaction } \\
(\times \mathrm{ppm})\end{array}$ & $\begin{array}{c}\text { Seed weight/ } \\
\text { plant }(\mathrm{g})\end{array}$ & $\begin{array}{l}\text { Seed yield } \\
(\mathrm{kg} / \mathrm{ha})\end{array}$ \\
\cline { 2 - 7 } & Pot & Field & Field & & Pot & Field & Field \\
\hline & & & & $\mathrm{V}_{1} \times 0$ & $7.51 \mathrm{c}$ & $8.23 \mathrm{~cd}$ & $1728 \mathrm{bc}$ \\
25 & $6.55 \mathrm{~d}$ & $7.28 \mathrm{~d}$ & $1556 \mathrm{c}$ & $\mathrm{V}_{1} \times 25$ & $7.97 \mathrm{~b}$ & $8.91 \mathrm{~b}$ & $1870 \mathrm{ab}$ \\
50 & $7.08 \mathrm{c}$ & $8.70 \mathrm{~b}$ & $1696 \mathrm{~b}$ & $\mathrm{~V}_{1} \times 50$ & $9.23 \mathrm{a}$ & $9.77 \mathrm{a}$ & $2052 \mathrm{a}$ \\
75 & $8.16 \mathrm{a}$ & $9.31 \mathrm{a}$ & $1893 \mathrm{a}$ & $\mathrm{V}_{1} \times 75$ & $7.99 \mathrm{~b}$ & $9.70 \mathrm{a}$ & $2037 \mathrm{a}$ \\
100 & $7.66 \mathrm{~b}$ & $8.70 \mathrm{~b}$ & $1913 \mathrm{a}$ & $\mathrm{V}_{1} \times 100$ & $7.74 \mathrm{~b}$ & $8.70 \mathrm{bc}$ & $1827 \mathrm{~b}$ \\
F test & $7.09 \mathrm{c}$ & $8.26 \mathrm{c}$ & $1704 \mathrm{~b}$ & $\mathrm{~V}_{2} \times 0$ & $5.58 \mathrm{e}$ & $5.99 \mathrm{~g}$ & $1384 \mathrm{~d}$ \\
Variety & $* *$ & $* *$ & $* *$ & $\mathrm{~V}_{2} \times 25$ & $6.18 \mathrm{~d}$ & $6.59 \mathrm{fg}$ & $1522 \mathrm{~d}$ \\
BINA mung-7 & $8.09 \mathrm{a}$ & $8.80 \mathrm{a}$ & $1902 \mathrm{a}$ & $\mathrm{V}_{2} \times 75$ & $7.32 \mathrm{c}$ & $7.74 \mathrm{de}$ & $1788 \mathrm{~b}$ \\
BINA mung-8 & $6.52 \mathrm{~b}$ & $7.60 \mathrm{~b}$ & $1601 \mathrm{~b}$ & $\mathrm{~V}_{2} \times 100$ & 6.43 & $6.84 \mathrm{f}$ & $1580 \mathrm{~cd}$ \\
\hline F test & $* *$ & $* *$ & $* *$ & & $*$ & $*$ & $*$ \\
\hline CV (\%) & 4.52 & 3.89 & 6.22 & & 3.89 & 4.52 & 6.22 \\
\hline
\end{tabular}

In a column, within treatment, figures having same letter(s) do not differ significantly at $\mathrm{p} \leq 0.05$.

$*, * *$ indicate significant at 5 and $1 \%$ level of probability; $\mathrm{V}_{1}=$ BINAmung- $7, \mathrm{~V}_{2}=$ BINAmung- 8 .

The highest seed yield of BINAmung-7 was recorded at $50 \mathrm{ppm}(9.23$ and $9.77 \mathrm{~g} / \mathrm{plant}$ for pot and field conditions, respectively) which was statistically similar to $75 \mathrm{ppm}$ chitosan (7.99 and $9.70 \mathrm{~g} / \mathrm{plant}$ for pot and field conditions, respectively). On the other hand, the highest seed yield of BINAmung-8 was at $75 \mathrm{ppm}$ (7.32 and $7.74 \mathrm{~g} / \mathrm{plant}$ for pot and field conditions, respectively) which was statistically similar to $50 \mathrm{ppm}$ chitosan $(7.09$ and $7.50 \mathrm{~g} / \mathrm{plant}$ for pot and field conditions, respectively). Result revealed that BINAmung-8 (bold seeded plant) were more influenced by chitosan application in seed yield (maximum $31.2 \%$ yield increased over control) than BINAmung-7, the small seeded variety (maximum $22.9 \%$ yield increased over control). And 50 and $75 \mathrm{ppm}$ had superiority for plant growth, yield components and fruit yield over 25 and 100 ppm.

\section{References}

Chibu H, Shibayama H and Arima S 2002. Effects of chitosan application on the shoot growth of rice and soybean. Japn. J. Crop Sci. 71: 206-211.

El-Tantawy EM 2009. Behaviour of tomato plants as affected by spraying with chitosan and aminofort as natural stimulator substances under application of soil organic amendments. Pakistan J. Biol. Sci. 12: 1164-1173.

Fakir MSA, Mondal MMA, Ismail MR and Ashrafuzzaman M 2011. Flower production and reproductive efficiency in mungbean. Int. J. Agric. Biol. 13: 966-970.

FAO 2007. Production Year Book of 2006. No. 67. Food and Agriculture Organization (FAO), Rome, Italy. p. 54 . 
Gornik K, Grzesik M and Duda BR 2008. The effect of chitosan on rooting of gravevine cuttings and on subsequent plant growth under drought and temperature stress. J. Fruit Ornamental Plant Res. 16: 333343.

Imam MH, Mondal, MMA, Prodhan AKMA, Malek MA and Islam MO 2010. Effect of foliar application of miyobi hormone on morpho-physiological attributes and yield in summer tomato. J. Agrofor. Environ. 4: 197-200

Khan WM, Prithiviraj B and Smiyh DL 2002. Effect of foliar application of chitin oligosaccharides on photosynthesis of maize and soybean. Photosynthetica 40: 621-624.

Mondal MMA, Puteh AB, Malek MA, Ismail, MR and Ashrafuzzaman M. 2012. Effect of foliar application of chitosan on growth and yield in okra. Aus. J. Crop Sci. 6: 918-921.

Nahar BS and Ikeda T 2002. Effect of silver-sheet and figaron on flower production, abscission of reproductive organ, yield and yield components in soybean. J. Agron. Crop Sci. 188: 193-200.

Patkowska E, Pieta D and Pastucha H 2006. The effect of biochikol 020 pc on microorganisms communities in the rhizosphere of Faba bean plants. Polish Chitin Sco. Monograph 11: 171-178.

Sereih A, Neven A, Abd-El-Aal S and Sahab AF. 2007. The mutagenic activity of chitosan and its effect on the growth of Trichoderma harzianum and Fuzarium oxysporum. J. Appl. Sci. Res. 3: 350-455.

Yoshida S, Forno DA, Cock JA and Gomes KA 1976. Laboratory manual for physiological studies of rice. 3rd ed., IRRI, Los Banos, Philippines.

(Manuscript received on 22 January, 2012; revised on 10 April, 2013) 